\title{
VDMOSFET MODEL PARAMETER EXTRACTION BASED ON ELECTRICAL AND OPTICAL MEASUREMENTS
}

\author{
C.-T. SALAME*, C. RIZK and G. JELIAN \\ Radiation Technology, Interfaculty Reactor Institute, Delft University \\ of Technology, Mekelweg 15, $2629 \mathrm{JB}$ Delft, The Netherlands
}

(Recetved 22 August 2000; In final form 13 September 2000)

\begin{abstract}
Lateral Device parameters for VDMOSFET (Vertical double diffused metal oxide semiconductor field effect transistor) with hexagonal cells has been extracted by an original model based on electrical and optical measurements. Using microscopic observation for the ship of the device and by C-V characterization, the lateral device atructure parameters could be extracted. Values of the extracted parameters are in good correlation with the values given by the manufactured. Advantage of this model the high precision results with a low cost. Perhaps, the most important point in this model, that we can replace the other techniques usually employed in this study where we destroyed the device for cut and cross section of the structure. The proposed technique in this paper can be very useful for analyzing any complex geometrical structure of VDMOS transistors (hexagonal, triangular, square etc...).
\end{abstract}

Keywords: VDMOSFET; Parameter extraction; Lateral parameters; Hexagonal cell

\section{INTRODUCTION}

The n-channel MOSFETs are the most popular for use in power switching circuits and applications. Various manufacturers produce power MOSFETs under the names HEXFET (International rectifier), VMOS (Phillips), SIPMOS (Siemens) and all consist of various physical designs diffused into an epitaxial substrate in multiple parallel

*Corresponding author. Tel.: +31 15278 3776, Fax: +31 15278 6442, e-mail: salame@ieee.org 
configurations [1-3]. VDMOSFET with hexagonal cells (HEXFET) has a complex geometrical structure. The size of these components is specially adapted by the manufacturers in response to the needs of the power [4-7]. Technical progress of microelectronic integration involve also an evolution towards the miniaturization which requires new structural modifications to answer the sub-micronics parasitic effects [8-11].

Lateral parameters of these devices are needed for software simulation models for the components structure. In order to describe our methodology, electrical measurements of the capacitance voltage relationship at a high frequency and optical measurements are performed.

The VDMOSFET structure and the measurements techniques are discussed in part 2. Part 3 deals with the extraction methodology based on the capacitance- voltage characterization and the microscopic observation.

\section{DEVICE STRUCTURE AND MEASUREMENTS TECHNIQUES}

An approximation of the internal structure of an VDMOSFET is shown in Figure 1. Drain and source connections are made to higher conduction high doped regions. The metal gate is electrically isolated from the p-type substrate by a layer of non-conducting silicon oxide $\left(\mathrm{SiO}_{2}\right)$. When a positive voltage is applied to the gate with respect to the source an electric field will be created pointing away from the base and across the p-region directly under the base. The electric field will cause positive charges in the p-region to move away from the base inducing or enhancing a n-region in its place. Conduction can then take place between the $n^{+}$(drain) $n$ (enhanced region) $n^{+}$(source). Increasing or decreasing the gate voltage will cause the induced $n$ channel to grow or decrease in size thus controlling conduction. The drive voltage or voltage applied between gate and source to switch the MOSFET ON must exceed a threshold value of $V_{\text {th }}$. Reducing the drive voltage to below $V_{\text {th }}$ will cause the MOSFET to turn OFF.

The equivalent circuit of an enhancement MOSFET is shown in Figure 2a. Two parasitic capacitance's between gate to source and gate 


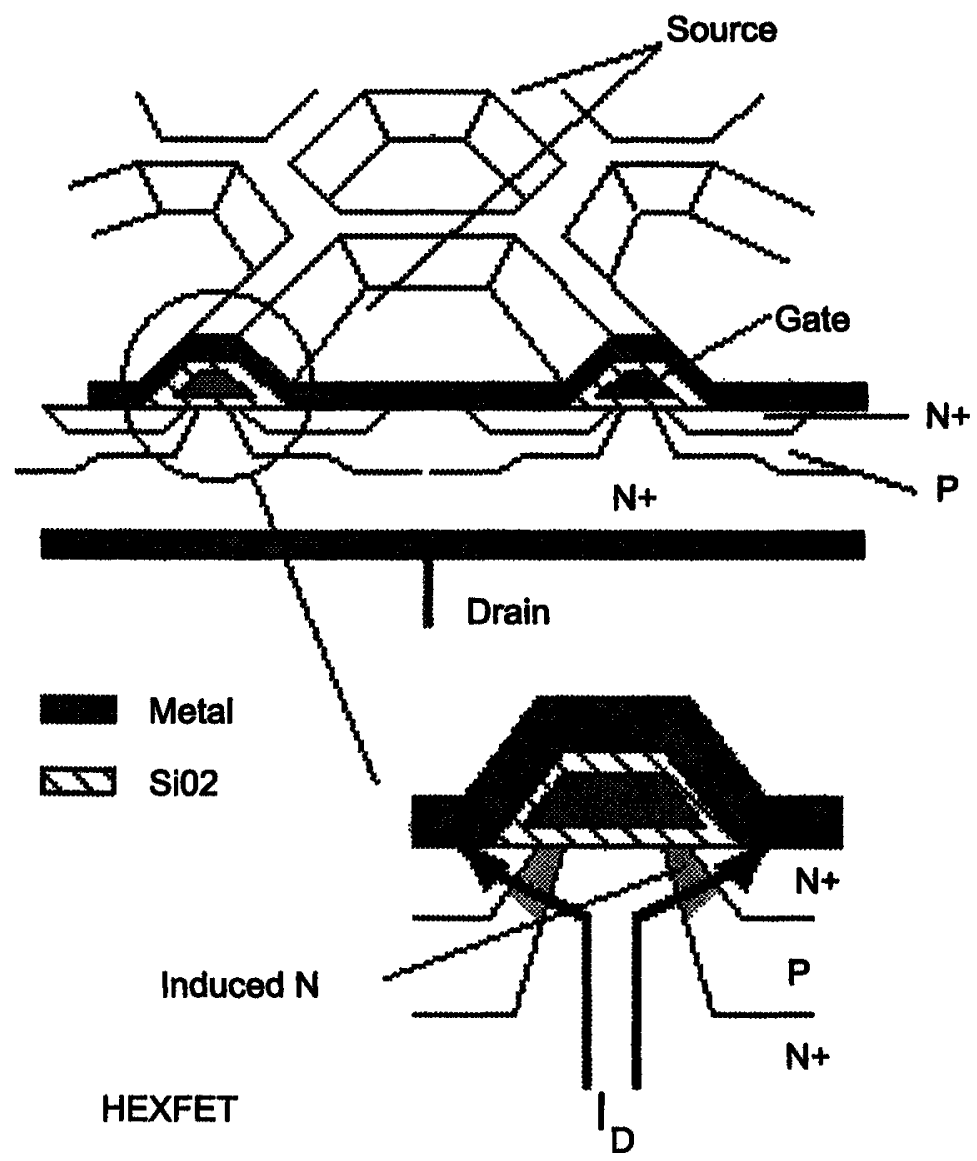

FIGURE 1 Approximation of the internal structure of an VDMOSFET.

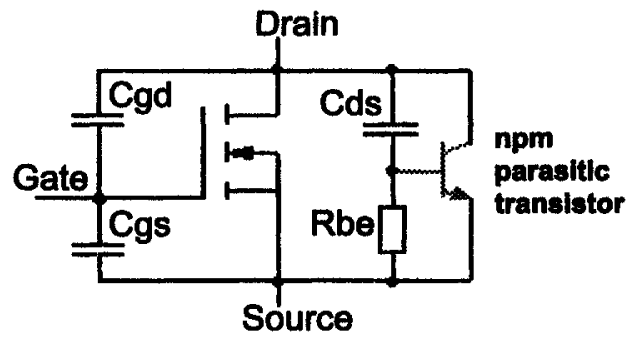

FIGURE 2a The equivalent electrical circuit of an enhancement VDMOSFET. 
to drain will cause switching delays if the gate driver cannot support large initial currents. A further parasitic capacitance and bipolar npn transistor exist between drain and source but due to the internal structure the transistor appears as a diode and capacitor connected between drain and source as shown in Figure $2 \mathrm{~b}$. Unfortunately the parasitic diode does not have the structure of a fast diode and must be neglected and a separate fast diode used in a high speed switching circuit.

The parallel connection of MOSFETs allows higher load currents to be handled by sharing the current between the individual switches. Because MOSFETs have a positive temperature coefficient they can be paralleled without the need for source resistors (BJTs need small emitter resistors that provide negative feedback). If one MOSFET starts to draw slightly more current than the others it heats up and its impedance increases which results in the current through it decreasing. Parallel MOSFETs should be mounted close together so that the gate drive impedances are the same and all transistors switch at the same time.

By a C-V measurement between the gate and the drain the capacitance's values for the $\mathrm{n}^{-}$-layer, the $\mathrm{n}$-channel and the overlap (source region $\mathrm{n}^{+}$under the gate oxide) can be determined. Microscopic observation for the device area is realized to determine the source $\left(\mathrm{L}_{\mathrm{s}}\right)$ and the gate $\left(\mathrm{L}_{\mathrm{g}}\right)$ lengths of one cell. In the optical measurements the gate length is the distance between two cells.

Combination between this two measurement is used to extract the different lateral parameters.

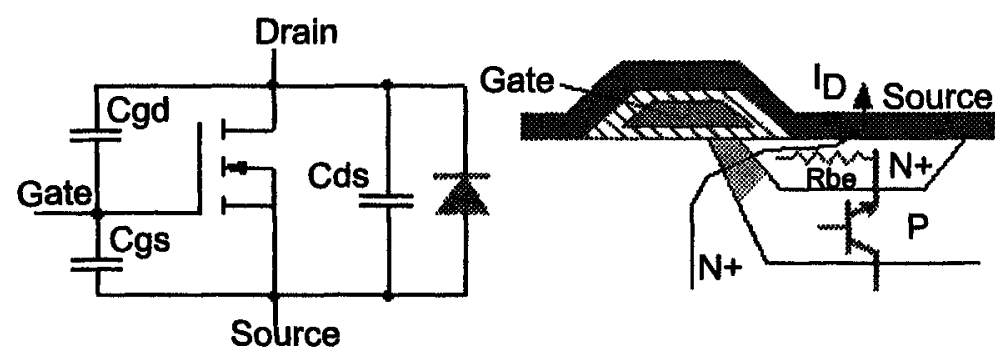

FIGURE $2 b$ The equivalent circuit of a VDMOSFET where the bipolar npn transistor appears as a diode and capacitor connected between drain and source. 


\section{EXTRACTION METHODOLOGY}

The cross-sectional view of the device is shown in Figure 3, the gate and the source lengths are determined by the microscopic observation. The capacitance measurement between the gate and the drain, $\mathbf{C}_{\mathbf{G D}}$, is used in order to extract the device parameters. The experimental set-up for the CV measurements is shown Figure 4. An HP4192A is used in the $\mathrm{CV}$ measurement. A small a.c voltage at $1 \mathrm{MHz}$ is applied between the High (gate) and the low (drain) potential electrodes. The capacitance through the grounded source electrode is not include in

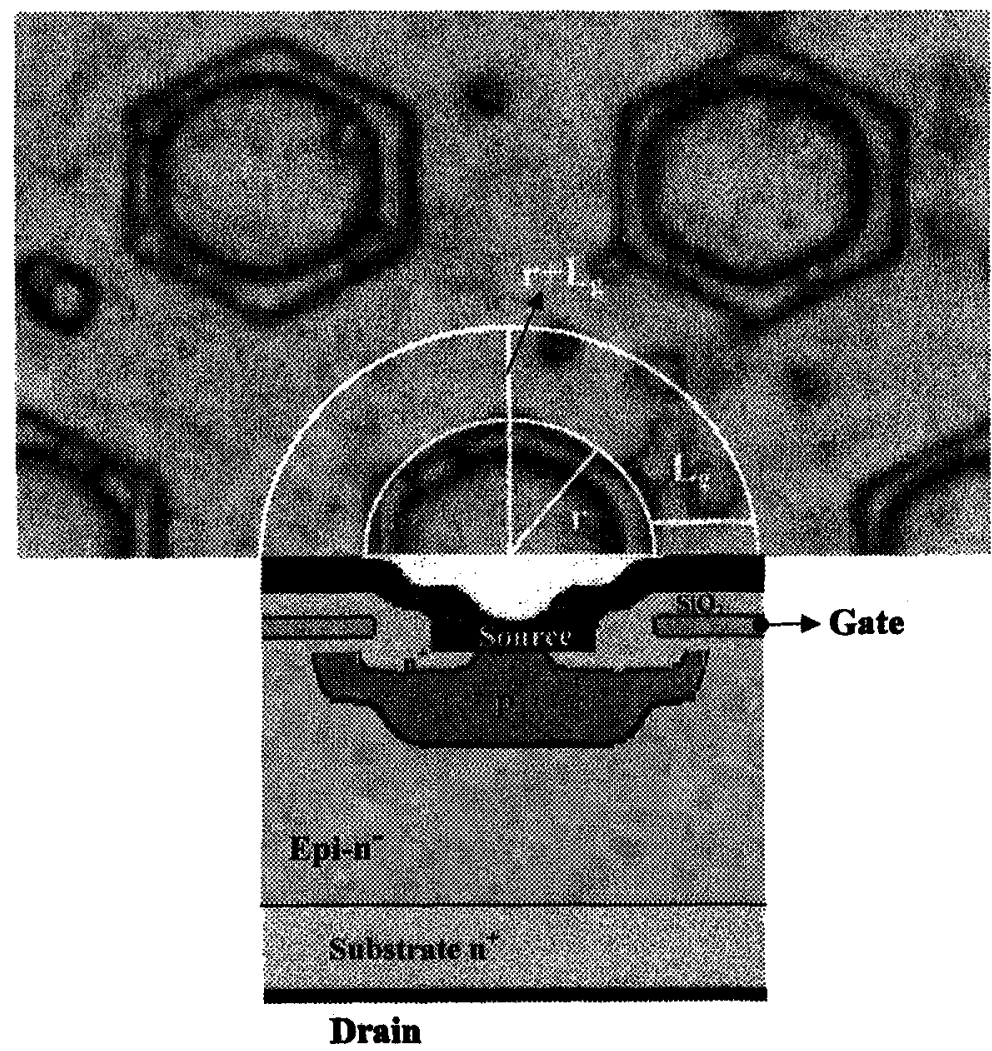

FIGURE 3 VDMOSFET Structure: top view and cut section. 


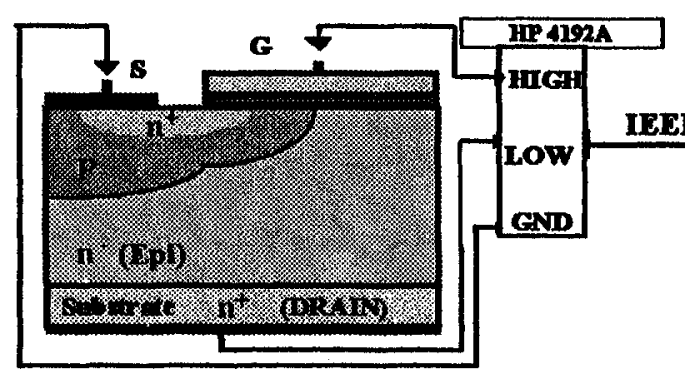

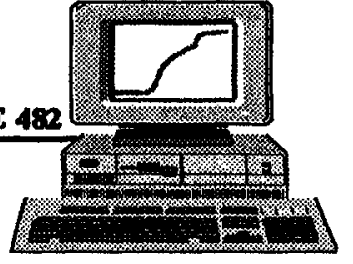

PC

FIGURE 4 The gate-drain capacitance $\left(\mathrm{C}_{O D}\right)$ measurement set-up.

the measurement because the small a.c voltage is not applied to the source electrode.

The measured capacitance $C_{G D}$ is shown in Figure 5 and the conceptional charge distributions with the applied d.c gate biases are shown in the Figure 6a, b.

When an the applied gate voltage is lower than the threshold voltage of $n^{-}$region $V_{T D}$, the $n^{-}$region, $p$ region and $n^{+}$region show inversion, accumulation and depletion modes, respectively (Fig. 6a). For this gate voltage values, the hole of the strongly inverted $n^{-}$region

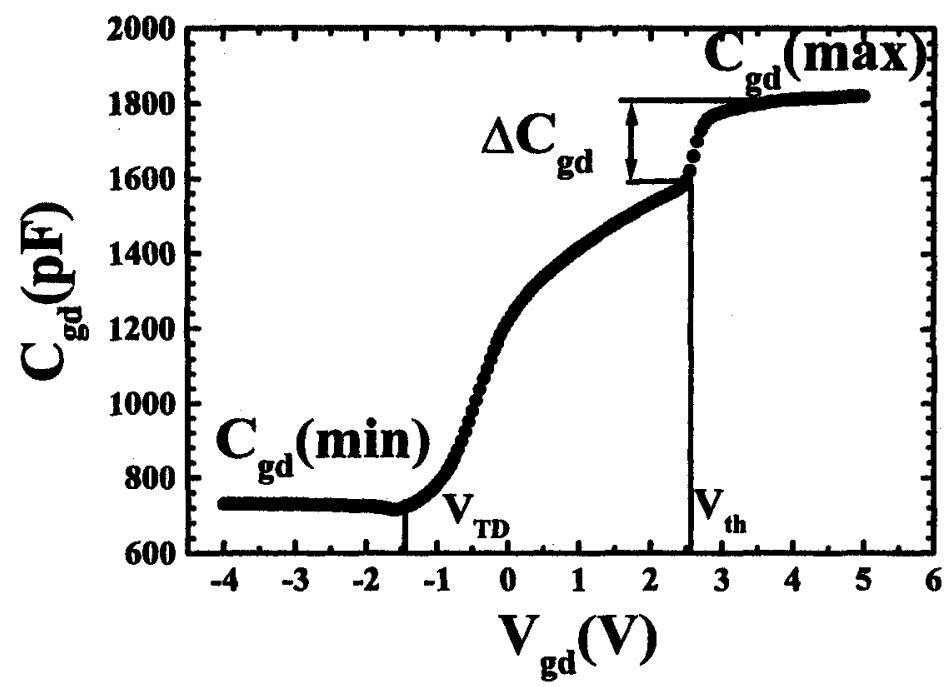

FIGURE 5 The measured gate-drain capacitance curve $\left(\mathbf{C}_{\mathbf{G D}}\right)$. 


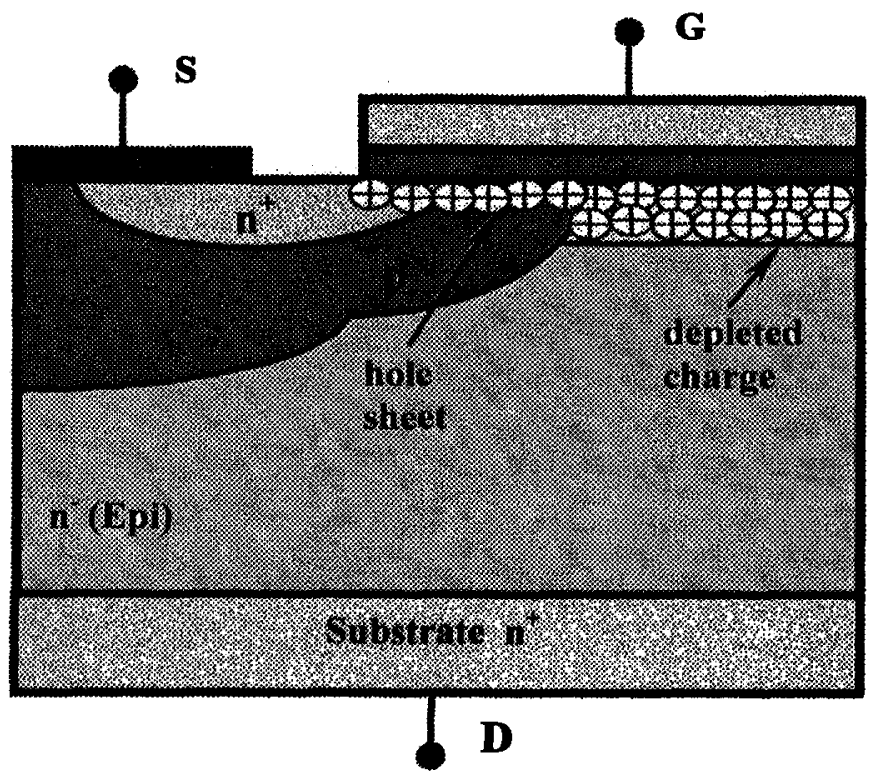

(a)

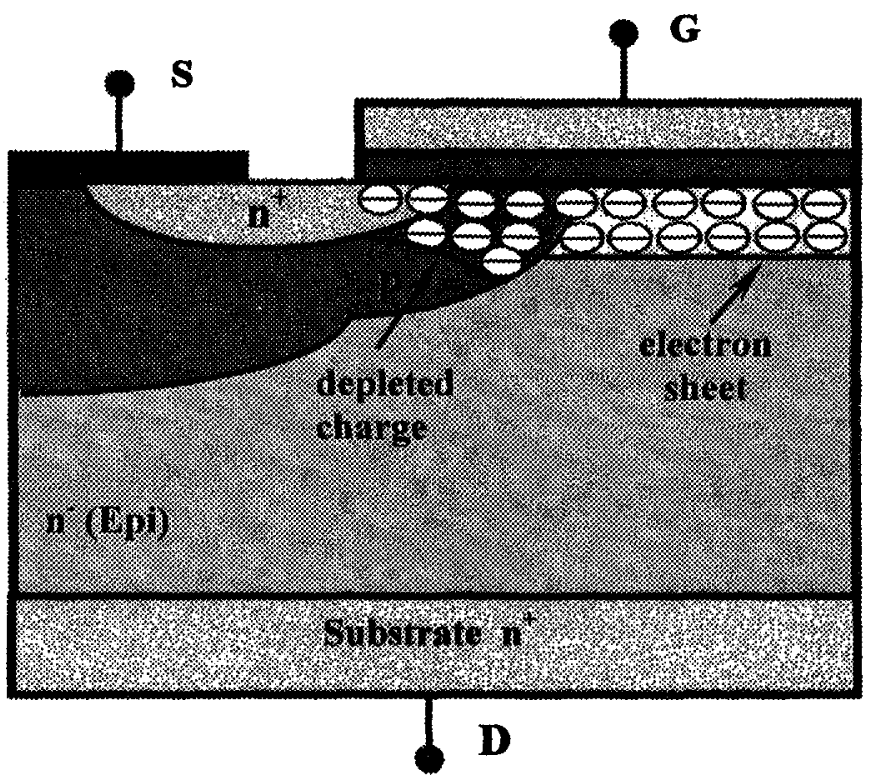

(b)

FIGURE 6 The conceptional charge distribution in VDMOSFET with the different applied gate biases: (a) $V_{G}<V_{T D}$ and (b) $V_{G}>V_{t h}$. 
cannot be supplied from the $p$ region (the $p$ region is floated at a. c). In this case, $C_{G D}$ is the minimum value consisted of metal-oxide- $n^{-}$ structure capacitance and its value $\mathbf{C}_{\mathrm{GD} \text {-min }}$ is written by:

$$
\frac{C_{G D-\min }}{N}=\frac{C_{O X} \cdot C_{D M}}{\left(C_{O X} \cdot C_{D M}\right) \cdot A_{n}^{-}}
$$

Where, $C_{o x}\left(=\varepsilon_{o x} / t_{o x}\right)$ is the gate capacitance per unit area, $A_{n}^{-}$is the area of the $n^{-}$region, $N$ the total number of the device cells, $C_{D M}$ is the depletion capacitance per unit area in the $\mathrm{n}^{-}$region given by:

$$
C_{D M}=\frac{\varepsilon_{s i}}{\sqrt{\left(2 \varepsilon_{s i} / q N_{D}\right) \psi_{D}}}
$$

where $\mathrm{N}_{\mathrm{D}}$ is the doping concentration of $\mathrm{n}^{-}$epitaxial layer, generally called the lightly doped drain region is about $3 \times 10^{14} \mathrm{~cm}^{-3}[12], \Psi_{D}$ is the surface potential in the strong inverted $\mathrm{n}^{-}$region.

Sine all the cells are mounted in parallel the unit cell capacitance are obtained dividing the measured device capacitance by the total number of cells $(\mathbf{N})$.

When the gate voltage is larger than $V_{\text {th }}$, which is the maximum threshold voltage of channel, the electrons in the accumulated $n^{+}$ region (Overlap) as well as the $p$ region (channel $n$ ) are supplied from the $\mathrm{n}^{-}$region (Fig. $6 \mathrm{~b}$ ). Therefore, $\mathrm{C}_{\mathrm{GD}}$ undergoes sudden increase at $V_{G S}=V_{\text {th }}$ and the capacitance variation at $V_{t h}, \Delta C_{G D}$, is the same as the oxide capacitance of the $\mathrm{n}^{+}$region written by:

$$
\Delta \mathrm{C}_{\mathrm{GD}} / \mathrm{N}=\mathrm{C}_{\mathrm{ox}} \cdot \mathrm{A}_{\mathrm{n} \text { (overlap) }}^{+},
$$

where $\mathrm{A}_{\mathrm{n} \text { (overlap) }}^{+}$is the area of the $\mathrm{n}^{+}$source region under the gate oxide. In the case of $V_{G S}>V_{t h}, C_{G D}$ is the same as the entire gate oxide capacitance and its value, $\mathrm{C}_{\mathrm{GD}(\max )}$, is represented by:

$$
\mathrm{C}_{\mathrm{GD}(\max )} / \mathrm{N}=\mathrm{C}_{\mathrm{OX}} \cdot \mathrm{A}_{\mathrm{gate}},
$$

where $A_{\text {gate }}$ is the total gate area.

The threshold voltage $V_{\text {th }}$ was determined in the saturation region where the drain current of the VDMOSFET is given [13] by:

$$
I_{D S(s a t)} \approx \mu_{n} C_{o x} W / L\left(V_{G s}-V_{t h}\right)^{2}
$$


By plotting $\sqrt{I_{D S}}$ versus $V_{G S}$ (for a given $V_{D S}>V_{D S(s a t)}$ ), the threshold voltage can be deduced from the linearly extrapolated value at the $V_{G S}$ axis (Fig. 7).

Structure of the unit hexagonal cell is approximate to cylinder (see Fig. 8) for the facility of the mathematical equations resolve. This approximation doesn't affect much the results precision since the parameters values determinate with our method have $96 \%$ of the values given by the device manufactured.

In the approximate unit cell of cylinder type, the total gate area $\mathrm{A}_{\text {gate, }}$ the $\mathrm{n}^{+}$source area (Overlap) $\mathrm{A}_{\mathrm{n}+\text { source }}$ and the $\mathrm{n}^{-}$region area $A_{n-}$ are represented by:

$$
\begin{gathered}
A_{g a t e}=\pi\left[\left(L_{s}+L_{g}\right)^{2}-L_{s}^{2}\right] \\
A_{n^{+} \text {source }}=\pi\left[\left(L_{s}+L_{n+\text { source }}\right)^{2}-L_{s}^{2}\right] \\
A_{n-}=\pi\left[\left(L_{s}+L_{g}\right)^{2}-\left(L_{s}+L_{n+\text { source }}+L_{c h}\right)^{2}\right] \\
\mathbf{L}_{g}=L_{n+\text { source }}+\mathrm{L}_{c h}+L_{n-}
\end{gathered}
$$

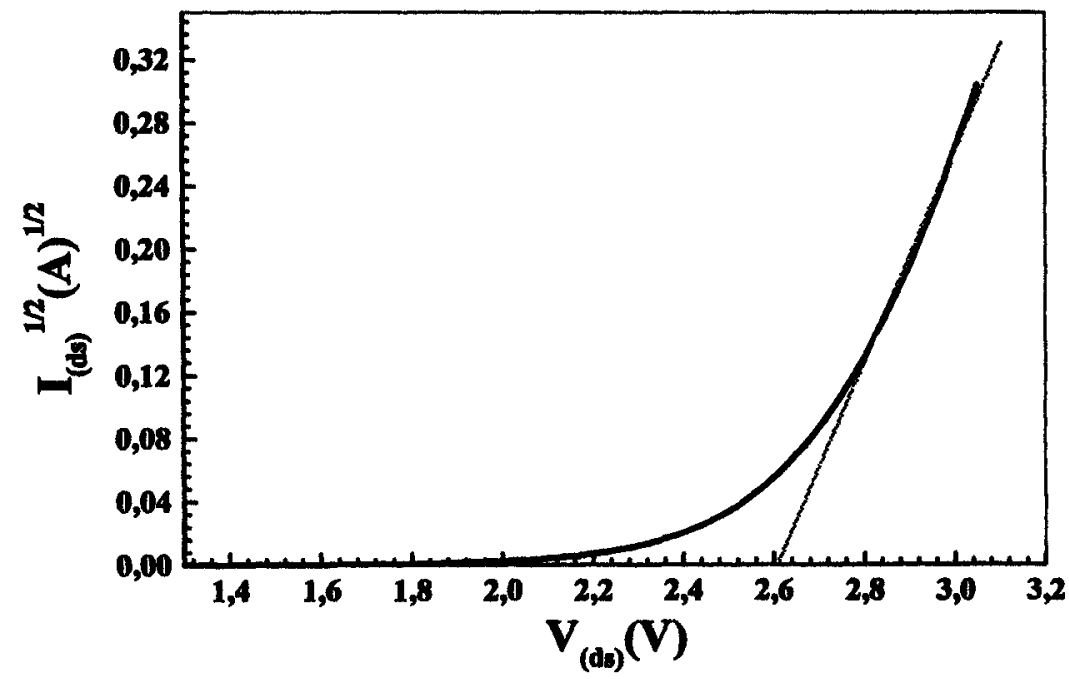

FIGURE 7 Determination of the threshold voltage of the VDMOSFET by electrical measurement. 


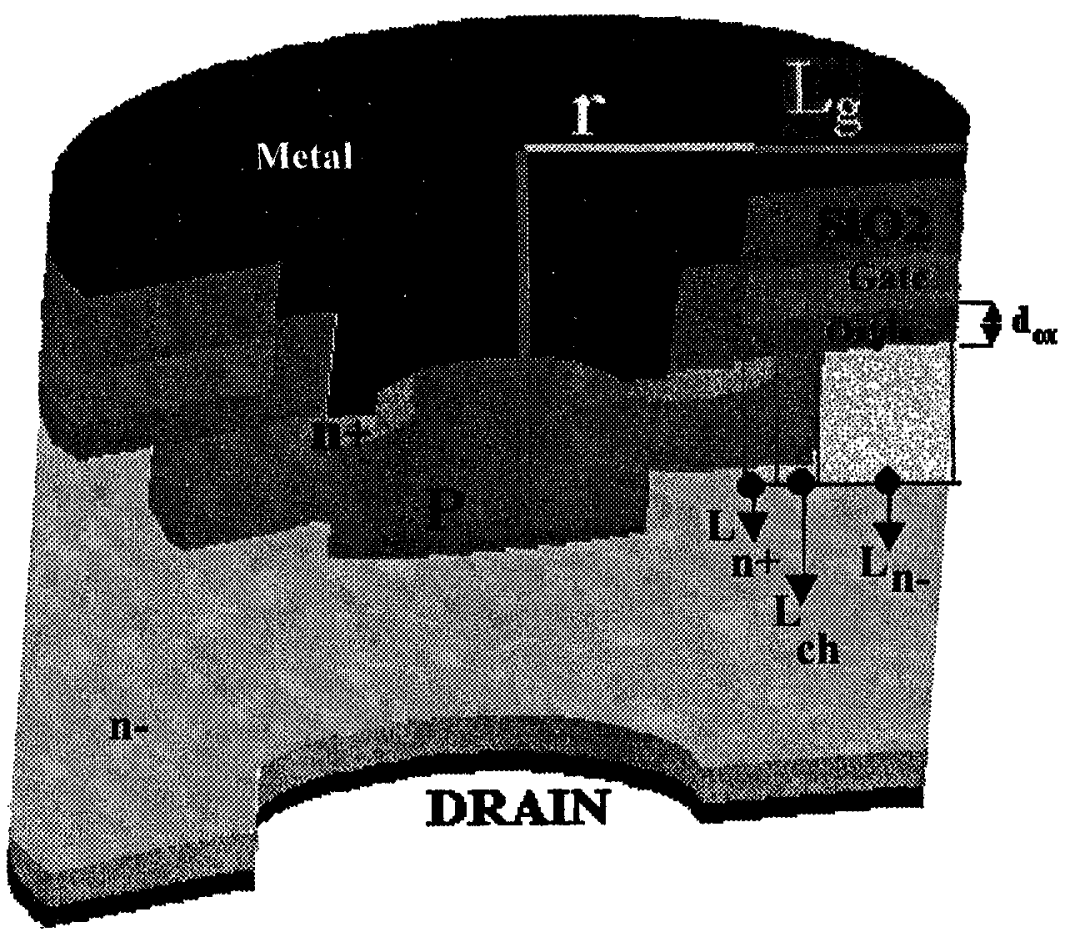

FIGURE 8 Structure of the unit hexagonal cell approximate to cylinder.

Where $r+L_{g}$ is the unit cell radius, $L_{n+\text { source }}$ is the $n^{+}$source length (Overlap), $L_{c h}$ is the channel length and $L_{n-}$ is the $n^{-}$region length. The total gate length $\left(\mathrm{L}_{\mathrm{g}}\right)$ is the sum of these length. $\mathrm{C}_{\mathrm{GD}(\max )}, \Delta \mathrm{C}_{\mathrm{GD}}$ and $C_{G D(m i n)}$ in the unit cell are $0.1698,0.0188$ and $0.0698 \mathrm{pF}$, respectively. From $C_{G D}(\max )$ and Eqs. (4) and (6), the gate oxide thickness of $98 \mathrm{~nm}$ is extracted and his value is in excellent agreement with the expected oxide thickness from the process $(100 \mathrm{~nm}) . \mathrm{L}_{n+\text { source }}$

TABLE I The comparison of the real dimensions and the extracted dimensions

\begin{tabular}{lcc}
\hline & Real lateral dimension & Extracted lateral dimension \\
\hline $\mathrm{d}_{\mathrm{ox}}$ & $100 \mathrm{~nm}$ & $98 \mathrm{~nm}$ \\
$\mathrm{~L}_{\mathrm{n}-}$ & 9 & 8,42 \\
$\mathrm{~L}_{\mathrm{ch}}$ & $1 \mu \mathrm{m}$ & $0,98 \mu \mathrm{m}$ \\
$\mathrm{L}_{\mathrm{n}+}$ & 0,7 & $0,6 \mu \mathrm{m}$ \\
\hline
\end{tabular}


is extracted from (3) and (7) and $L_{c h}$ is extracted from (1) and (8). Then $L_{n-}$ is extracted from the determined $L_{n+\text { sources }} L_{c h}$ and (9).

The device parameter lengths extracted using the propose method in this paper, are listed in Table $I$.

\section{CONCLUSION}

An original model for power MOSFETs lateral parameters extraction have been presented. Values of the extracted parameters are in excellent agreement with the values given by the devices fabricant. These parameters are very important for device structure simulation and modulation to evaluate degradation properties cased by electrical stress, radiation effects etc...

\section{References}

[1] Baliga, B. J. (1981). "Silicon Power Field Controlled Devices", Stlicon Integrated Circuits, Part B, Academic Press, New York, pp. 158-174.

[2] Baliga, B. J. (1982). "High-Voltage Device Terminaison Techniques-A Comparative Review", IEEE Proc., 129, 173-179.

[3] Baliga, B. J., "Modern Power Devices", John Wiley, New York, 1987.

[4] Gagnard, X., Taurin, M. and Bonnaud, O. (1999). "New rapid method for lifetime determination of gate oxide validated with Bipolar/CMOS/DMOS technology" ESREF99 Conference Proceeding, pp. 785-790.

[5] Arnould, J. et Merle, P., "Dispositifs de l'électronique de puissance", Traité des nouvelles technologies (Electronique), Hermes, Paris, 1992.

[6] Chang, H. R. and Baliga, J. (1987). "Impact of cell breakdown upon power DMOSFET on-resistance". IEEE Transactions on Electron Devices, ED-34.

[7] Goodenough, F., "Power MOSFET and bipolar technologies merge to get the best of both worlds", Electronic Design, pp. 53-56, juillet, 1983.

[8] "Power MOS Devides-data book"-1 1 ere 6 dition-SGS-THOMSON- juin, 1988.

[9] Rossel, P. (1984). "Power MOS Device", Microelectron, Reliab., 24, 339- 366.

[10] Lee, J. C., Chen, I.-C. and Hu, C., "Modelling and characterisation of gate oxide reliability", IEEE Transactions on Electron Devices, 35(12), December, 1988, 2268-2278.

[11] Cunningham, J. A., "The Use and Evaluation of Yield Models in Integrated Circuit Manufacturing", In: IEEE Trans. On Semiconductor Manufacturing, 3(2), 60-71.

[12] Chung-Min Liu and James B. Kuo (1995). "Turn-off Transient Analysis of Double Diffused Metal-Oxide-Semiconducteur Device Considering Quasi Saturation", Appl. Phys., 34, 869-873.

[13] Sze, S. M., Physics of Semiconductor Devices, Ch. 8, John Wiley, New York, 1981. 

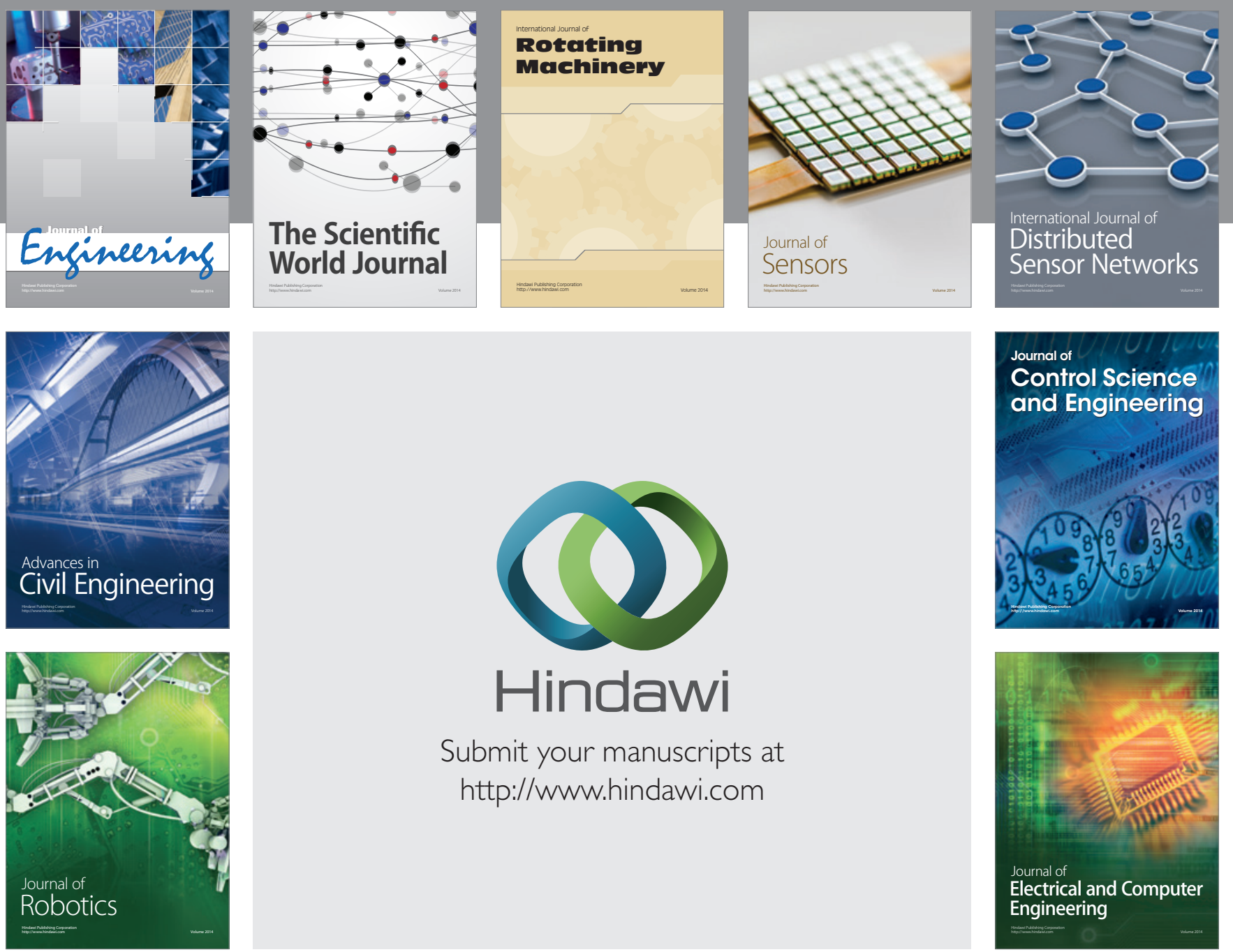

Submit your manuscripts at

http://www.hindawi.com
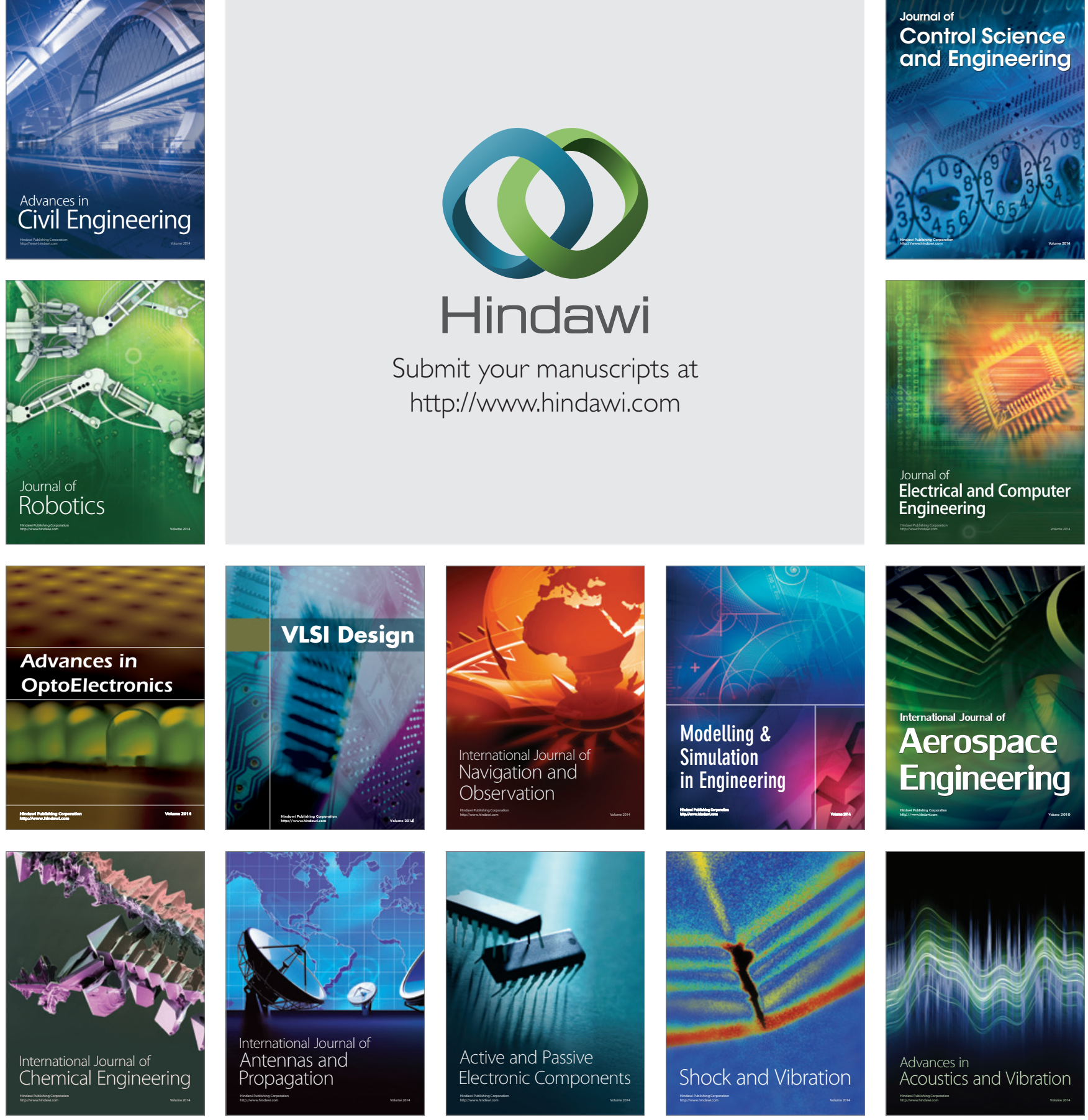